\title{
Exclude Organ Measurements Data From Statistics Indicator
}

National Cancer Institute

\section{Source}

National Cancer Institute. Exclude Organ Measurements Data From Statistics Indicator. NCl Thesaurus. Code C119885.

Specifies whether the organ measurement result values should be excluded from the statistical analysis. 